\title{
Existence of Positive Solutions for Semilinear Elliptic Equations in General Domains
}

\author{
Joel SMOller \& ARThur WASSERMAN
}

To James Serrin for his sixtieth birthday

\section{§ 1. Introduction}

The main purpose of this paper is to prove some new existence theorems for positive solutions to the Dirichlet problem

$$
\begin{gathered}
\Delta u(x)+f(u(x))=0, \quad x \in \Omega, \\
u(x)=0, \quad x \in \partial \Omega .
\end{gathered}
$$

Here $\Omega$ is a bounded domain in $\mathbb{R}^{n}$, with smooth boundary, and $f$ is a continuous function on $\mathbb{R}$.

We introduce in $\S 2$ the notion of "eccentricity", $e(\Omega)$, of a domain $\Omega \subset \mathbb{R}^{n}$, which has the property that $1 \leqq e(\Omega)<\infty$ and $e(\Omega)=1$ if and only if $\Omega$ is a ball. We then define for any function $f$ on $\mathbb{R}$ the "nonlinearity" of $f, N(f)$. If $f$ is a linear function, $N(f)=1$; if $f(u)=u^{k}, k<1, N(f)=\infty$; if $f(u)=u^{k}$, $k>1, N(f)<1$. The main theorem of the paper, Theorem 2.2, states that if $N(f)>e(\Omega)$ then there exist positive solutions to $(1.1),(1.2)$ on all domains $\lambda \Omega$ if $\lambda$ is sufficiently large. Equivalently, if $N(f)>e(\Omega)$, then positive solutions to the Dirichlet problem for $\Delta u+\mu f(u)=0$, exist on $\Omega$, for some range of $\mu$. Our method of proof is a variation of the technique of "upper and lower solutions", $c f .[14$, Ch. 10], whereby we use techniques developed in [10, 13] (where $\Omega$ is an $n$-ball) in order to construct these "solutions". We note that our methods apply to any elliptic operator; we consider (1.1) for definiteness. We then give in $\S 3$, several applications of this theorem. For example, if $f(u) / u \rightarrow 0$ as $u \rightarrow \infty$, and $^{1} \quad F\left(u_{0}\right)>0$ for some $u_{0}>0$ (where $F^{\prime}=f, F(0)=0$ ) then positive solutions exist on $\lambda \Omega$ if $\lambda$ is sufficiently large. Again, if $f\left(u_{0}\right)=0$ for some $u_{0}>0$, and $F\left(u_{0}\right)>0$, then the same conclusion holds. In both cases, we require no conditions on the behavior of $f$ near zero.

In $\S 4$ we take $\Omega$ to be an $n$-ball, and we prove existence of positive solutions

1 This is always a necessary condition as follows from PoHozaEv's identity; see [2] and [9]. 
of (1.1), (1.2) on some ball, provided only that $f$ satisfies an inequality of the form

$$
c_{1} \leqq f(u) / u^{k} \leqq c_{2} \quad \text { as } u \rightarrow \infty
$$

Here $c_{1}$ and $c_{2}$ are positive constants, and $0<k<n /(n-2)$. This result is a consequence of some general existence theorems whereby $f$ is required to satisfy an inequality of the form $c u^{\alpha} \leqq f(u) \leqq d u^{\beta}$, for large $u$, with $c, d, \alpha$ and $\beta$ being positive constants.

In $\S 5$ we study the Neumann problem for (1.1) on $n$-balls, i.e. solutions of (1.1) which satisfy homogeneous Neumann boundary conditions

$$
d u(x) / d n=0, x \in \partial \Omega,
$$

where $d / d n$ denotes differentiation in the radial direction on $\partial \Omega$. Thus, for example, if $f$ satisfies (1.3), where $0<k<n /(n-2)$, and, in addition, there exists an $\eta$ such that $f(u)<0$ for $u \leqq \eta<0$, and $F(u) \rightarrow \infty$ as $u \rightarrow-\infty$, we show that the Neumann problem has a monotone radial solution on some $n$ ball.

We note that the existence of positive solutions to the Dirichlet problem has been obtained if $f(u)$ is required to be positive, or if $f(0)=0$ and $f^{\prime}(0)>0$; see $[1,2,3,5,7,8]$. We allow $f$ to be an arbitrary function near zero. Such freedom is necessary if one is interested in questions of "symmetry-breaking" (see $[11,12])$. Indeed, as we have shown in [11], the symmetry cannot break on a positive radial solution of (1.1), (1.2) unless $f(0)<0$.

We remark that if $f(u)=u^{k}$, then it is well known [8] that there are no positive solutions to the Dirichlet problem on $n$-balls, if $k \geqq \frac{n+2}{n-2}=\frac{n}{n-2}+\frac{2}{n-2}$, but that there are such sclutions if $k<\frac{n+2}{n-2}$; see $[7,9]$. Thus our result given above is probably not the best possible. It would be interesting to know if there are positive solutions to the Dirichlet problem on $n$-balls if $f(u)=u^{k}-\sigma$, where $\sigma$ is a positive constant, and $\frac{n}{n-2}<k<\frac{n+2}{n-2}$.

Throughout this paper, $F$ will denote the primitive of $f$ satisfying $F(0)=0$, and $D_{R}^{n}$ will be the $n$-ball of radius $R$ centered at $0 \in \mathbb{R}^{n}$. Finally prime, ('), will denote differentiation with respect to $r$.

\section{§ 2. The Dirichlet Problem on General Domains}

In this section we shall show that the existence of positive solutions on $n$-balls can be used to prove the existence of positive solutions on general domains. We introduce the notion of the eccentricity of a domain, and we use it to prove our general, (somewhat abstract), existence theorem. In the next section we shall how it applies to extend known results.

Thus let $\Omega$ be a smooth bounded domain in $\mathbb{R}^{n}$, and let $B_{r}(p)$ denote an $n$ ball of radius $r$ centered at $p$. We begin with a purely geometrical result. 
Lemma 2.1. There exists $a \varrho>0$ such that if $x \in \Omega$, then $x \in \overline{B_{e}(y)} \subset \Omega$ for some $y \in \Omega$.

Proof. We start by mimicking the standard proof of the tubular neighborhood theorem; $c f$. [6]. Thus define a function $\phi: \partial \Omega \times \mathbb{R} \rightarrow \mathbb{R}^{n}$ by

$$
\phi(p, t)=p+t \bar{n},
$$

where $\bar{n}$ is the outward normal at $p$. Since $d \phi(p, 0)$ is invertible, $\phi$ is a local diffeomorphism. But $\phi$ is a diffeomorphism on the compact manifold $\partial \Omega \times\{0\}$; thus $\phi$ is, in fact, a diffeomorphism on some closed neighborhood of $\partial \Omega$ in $\partial \Omega \times \mathbb{R}$ (see [6]). Let $\partial \Omega \times[-\varrho, \varrho]$ be such a neighborhood, $\varrho>0$. We claim that this $\varrho$ "works". Thus, let $x \in \Omega$. If $x \notin \phi(\partial \Omega \times(-\varrho, 0])$, then $\overline{B_{Q}(x)} \subset \Omega$; otherwise we could find $z \in B_{0}(x) \cap \partial \Omega$ with $x=z+t \bar{n}$ for some $t,|t|<\varrho$, (namely $z$ is the point on $\partial \Omega$ closest to $x$ ), and so $x \in \phi(\partial \Omega \times(-\varrho, 0])$. On the other hand, if $x \in \phi(\partial \Omega \times(-0,0])$, then $x=p+t \bar{n}$ for some $p \in \partial \Omega$, and $-\varrho<t \leqq 0$. It follows that $x \in \overline{B_{0}(p-\varrho \bar{n})}$, and the proof is complete.

Note that lemma 2.1 requires $\Omega$ to have a smooth boundary; if $\partial \Omega$ has a cusp, then $\varrho=0$.

In view of this lemma, we may define the number

$$
\varrho(\Omega)=\sup \left\{\varrho>0: x \in \Omega \Rightarrow \exists y \in \Omega \text { with } \overline{B_{Q}(y)}<\Omega\right\} .
$$

Next, we define the eccentricity, $e(\Omega)$, of the region $\Omega$ by

$$
e(\Omega)=\inf \left\{\frac{R}{\varrho(\Omega)}: \Omega \subset \overline{B_{R}(p)} \text { for some } p \in \mathbb{R}^{n} \text {, and some } R>0\right\} .
$$

Observe that $e(\Omega) \geqq 1$, and $e(\Omega)=1$ if and only if $\Omega$ is an $n$-ball. To get a feel for $e(\Omega)$, note that if $\Omega \subset R^{2}$ is the ellipse $a^{2} x^{2}+b^{2} y^{2} \leqq a^{2} b^{2}$, where $b>a$, then $\varrho(\Omega)=a^{2} / b$, and since $\Omega \subset \overline{B_{b}(0,0)}$, we see that $e(\Omega)=b^{2} / a^{2}$.

Consider 4-tuples $\left(u_{1}, R_{1}, u_{2}, R_{2}\right)$ where $R_{1}, R_{2}>0$ and $u_{i}: D_{R_{i}}^{n} \rightarrow \mathbb{R}$. We say that a 4-tuple is admissible if there exist functions $f_{i}(u)$ with $f_{1}(u) \leqq f(u)$ $\leqq f_{2}(u)$ such that

i) $\Delta u_{i}(x)+f_{i}\left(u_{i}(x)\right)=0 \quad x \in D_{\mathrm{R}_{i}}^{n}$

ii) $u_{1}(x)=0$ and $u_{1}^{\prime}(x)<0 \quad$ if $|x|=R_{1}$

iii) $u_{2}(x) \geqq u_{1}(y) \geqq 0 \quad$ for all $x \in D_{R_{2}}^{n}, y \in D_{R_{1}}^{n}$.

Note that $u_{1}$ is a lower solution of 1.1 and $u_{2}$ is an upper solution of 1.1. See Figure 1. ( $D_{R}^{n}$ is the $n$-ball of radius $R$ centered at the origin.)

We define $N(f)$, the "nonlinearity" of $f$, by $N(f)=\sup \frac{R_{2}}{R_{1}}$ where the sup is taken over all admissible 4-tuples; if there is no admissible 4-tuple we set $N(f)=0$.

Remark. There exists an admissible 4-tuple for $f$ iff $F(p)>0$ for some $p>0$. We show (Lemma 3.1) that $F(p)>0$ for some $p>0$ is a sufficient condition 
for the existence of a pair $\left(u_{1}, R_{1}\right)$ satisfying i). and ii). We can complete this to an admissible 4-tuple by setting $f_{2}(u)=\sup \left(f(u): 0 \leqq u \leqq 2 u_{1}(0)\right\}$ and letting $u_{2}(r)$ be the radial solution of $\Delta u+f_{2}(u)=0$ satisfying $u_{2}(0)=2 u_{1}(0)$. If we define $R_{2}$ by $u_{2}\left(R_{2}\right)=u_{1}(0)$ we have that $\left(u_{1}, R_{1}, u_{2}, R_{2}\right)$ is an admissible 4-tuple and $N(f)>0$.
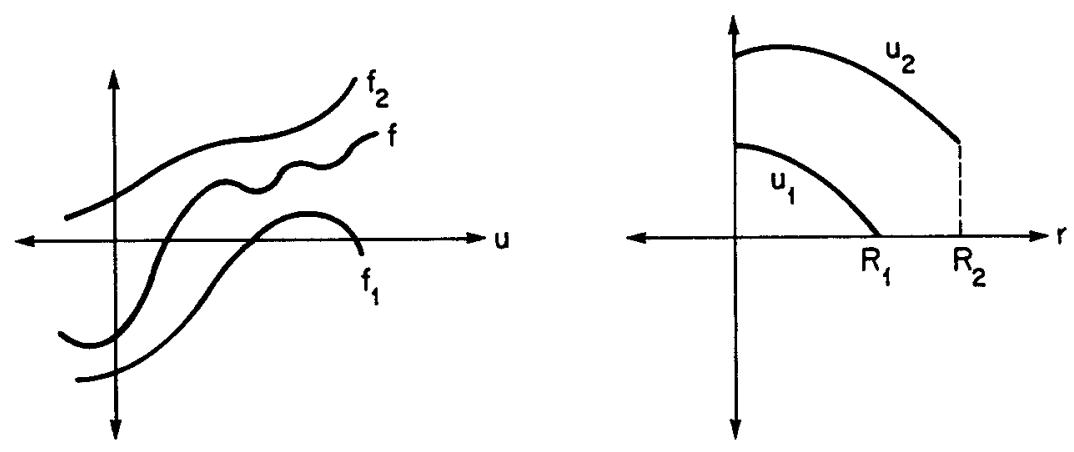

Fig. 1

On the other hand the condition $F(p)>0$ for some $p>0$ is a necessary condition for the existence of positive solutions of (1.1), (1.2); see [2]. Thus if $u\left(x_{1}\right)=\max \{u(x): x \in \Omega\}$, then $\Delta u\left(x_{1}\right)<0$ so $f\left(u\left(x_{1}\right)\right)>0$. The well-known Pohozaev identity (see [9]),

$$
2 n \int_{\Omega} F(u) d x-(n-2) \int_{\Omega} u f(u) d x=\int_{\partial \Omega}\left(\frac{d u}{d n}\right)^{2}(r \cdot n) d s,
$$

implies that $F$ must be positive on some ball centered at $x_{1}$. Thus, if $\left(u_{1}, R_{1}, U_{2}, R_{2}\right)$ is an admissible 4-tuple, $F_{1}(p)>0$ for some $p>0$ and $F(p) \geqq F_{1}(p)>0$. Hence, there exists an admissible 4-tuple if and only if $F(p)>0$ for some $p>0$ if and only if $N(f)>0$.

To gain some feeling for $N(f)$ we sketch an argument to show that $N(f)=$ 1 if $f(u)=\gamma u, \gamma>0$.

If $u(r)$ is a radial solution to (1.1),(1.2) on $\Omega=D_{R}^{n}$ set $f_{1}=f_{2}=f, u_{1}=u$, $u_{2}=c u, R_{1}=R$ and define $R_{2}$ by $u_{2}\left(R_{2}\right)=u_{1}(0)$ i.e. $c u\left(R_{2}\right)=u(0)$. Note that $R_{2}<R=R_{1}$ but as $c \rightarrow \infty, R_{2} \rightarrow R$, and hence $R_{2} / R_{1} \rightarrow 1$ and $N(f) \geqq 1$.

Next we show $N(f) \leqq 1$. Suppose $\left(u_{1}, R_{1}, u_{2}, R_{2}\right)$ is an admissible 4-tuple with $R_{2}>R_{1}$. Then $\Delta u_{1}+f_{1}\left(u_{1}\right)=0, \Delta u_{2}+f_{2}\left(u_{2}\right)=0$, and hence by multiplying the first equation by $u_{2}$, the second by $u_{1}$, subtracting and integrating over $D_{R_{1}}^{n}=\Omega$, we get $\int_{\Omega}\left(u_{2} \Delta u_{1}-u_{1} \Delta u_{2}\right)+\int_{\Omega} u_{2} f_{1}\left(u_{1}\right)-u_{1} f_{2}\left(u_{2}\right)=0$. Applying Green's theorem twice we get $\int_{\partial \Omega} u_{2} \frac{d u_{1}}{d n}+\int_{\Omega} u_{2} f_{1}\left(u_{1}\right)-u_{1} f_{2}\left(u_{2}\right)=0 ; \quad$ but $f_{1}\left(u_{1}\right) \leqq \gamma u_{1}$ and $f_{2}\left(u_{2}\right) \geqq \gamma u_{2}$ hence $u_{2} f_{1}\left(u_{1}\right)-u_{1} f_{2}\left(u_{2}\right) \leqq 0$. Also $u_{2}>0$ on $\partial \Omega$ and $\frac{d u_{1}}{d n}=u_{1}^{\prime}<0$ by ii). Thus $0=\int_{\partial} u_{2} u_{1}^{\prime}+\int_{\Omega} u_{2} f_{1}\left(u_{1}\right)-u_{1} f_{2}\left(u_{2}\right)<0$. Hence $\boldsymbol{R}_{1} \geqq \boldsymbol{R}_{2}$ and $N(f)=1$. 
With a little more effort one can show that the function

$$
f(u)=\left\{\begin{array}{ll}
\mu_{1} u & u \leqq 1 \\
\mu_{2}(u-1)+\mu_{1} & u \geqq 1
\end{array} \text { where } \mu_{1}>\mu_{2}>0 \text { has } N(f)=\sqrt{\frac{\mu_{1}}{\mu_{2}}}\right.
$$

Similarly the function $f(u)=u^{k}$ has $N(f)<1$ if $k>1$ and $N(f)=\infty$ if $k<1$ (see Theorem 3.3).

Theorem 2.2. If

$$
N(f)>e(\Omega),
$$

then there exists $\lambda>0$ and a positive solution to

$$
\begin{gathered}
\Delta u(x)+f(u(x))=0, \quad x \in \lambda \Omega, \\
u(x)=0, \quad x \in \partial(\lambda \Omega) .
\end{gathered}
$$

Equivalently if (2.3) holds, then there exists $\mu>0$ such that the problem

$$
\begin{gathered}
\Delta u(x)+\mu f(u(x))=0, \quad x \in \Omega \\
u(x)=0, \quad x \in \partial \Omega,
\end{gathered}
$$

has a positive solution.

Proof. We shall give the proof of statement i) since the two statements are easily seen to be equivalent through a simple scaling argument.

We first need the following lemma.

Lemma 2.3. Let $f \in C^{1}$, and let $u_{1}$, be a non-negative solution of the problem

$$
\begin{array}{ll}
u^{\prime \prime}+\frac{n-1}{r} u^{\prime}+f(u)=0, & 0<r<R \\
u(0)=p>0, \quad u^{\prime}(0)=0, & u\left(R_{1}\right)=0 .
\end{array}
$$

Suppose that $u^{\prime}\left(R_{1}\right)=q<0$. Then there exists an $\tilde{f} \in C^{1}$ such that the following hold:

(a) $\tilde{f}(u)=f(u)$ if $u \geqq 0$,

(b) If $\tilde{u}$ solves (2.3) with $\tilde{f}$ replaced by $f$, then $\tilde{u}^{\prime}(r)<0$ for all $r>0$ (so $\tilde{u}(r)<0$ if $\left.r>R_{1}\right)$.

Proof. Choose $B>0$ such that $f(0)>-B$. Then let $a>0$ satisfy

$$
\frac{3}{4} q^{2}>\frac{4(n-1)}{|q| R_{1}}\left(q^{2}+2 a B\right) a+2 a B
$$


note that this can be achieved for small $a>0$. Let $\tilde{f} \in C^{1}$ satisfy (cf. Figure 2):

i) $\tilde{f}(u)=f(u), \quad u \geqq 0$

ii) $|\tilde{f}(u)|<B, \quad u \leqq 0$

iii) $\tilde{f(u)} \equiv 0$ if $u \leqq-a$.

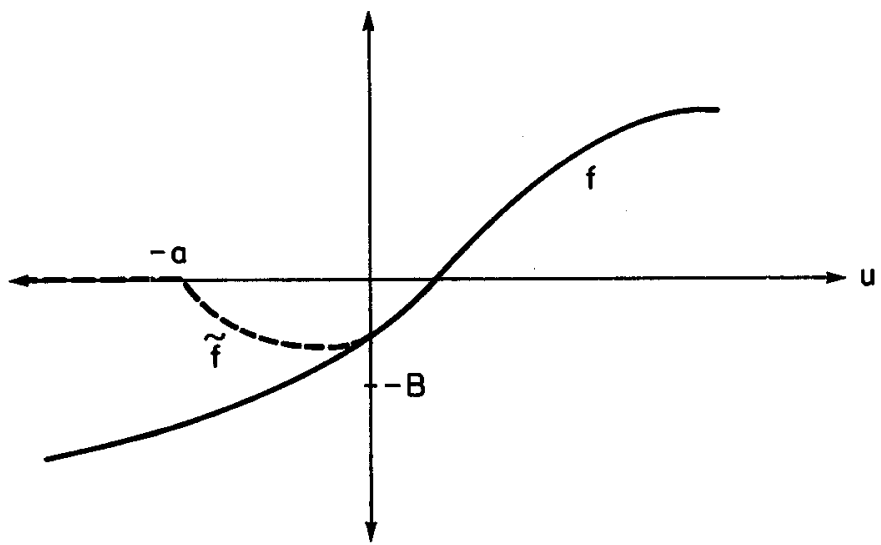

Fig. 2

We now verify that $\tilde{f}$ meets our requirement; i.e. $\tilde{u}^{\prime}(r)<0$ if $r>R_{\mathbf{1}}$. As a first step, we will prove that

$$
\tilde{u}^{\prime}(r)<q / 2 \quad \text { if } R_{1}<r \leqq R_{1}+\frac{2 a}{|q|} .
$$

Thus, set

$$
\tilde{F}(u)=\int_{0}^{u} \tilde{f}(s) d s
$$

then

$$
|\tilde{F}(u)| \leqq a B \quad \text { if }-a \leqq u \leqq 0 .
$$

We write, as usual, $H(u, v)=v^{2} / 2+\tilde{F}(u)$. Then since $H^{\prime}=-\frac{n-1}{r} v^{2} \leqq 0$, we see that for $r \geqq R_{1}, \tilde{v}^{2}+2 \tilde{F}(\tilde{u}) \leqq q^{2}$ (where $\tilde{v}=\tilde{u}^{\prime}$ ). Thus

$$
\tilde{v}(r)^{2} \leqq q^{2}+2 a B, \quad r \geqq R_{1} .
$$

But since

$$
\tilde{v}^{2}+2 \tilde{F}(\tilde{u})=q^{2}-2(n-1) \int_{R_{1}}^{r} \frac{\tilde{v}^{2}(t)}{t} d t,
$$


we have that

$$
\begin{aligned}
\tilde{v}^{2} & \geqq q^{2}-2 \tilde{F}(u)-2(n-1) \int_{R_{1}}^{r} \frac{q^{2}+2 a B}{R_{1}} d t \\
& =q^{2}-2 \tilde{F}(u)-2(n-1)\left(r-R_{1}\right) \frac{\left(q^{2}+2 a B\right)}{R_{1}} \\
& \geqq q^{2}-2 a B-2(n-1) \frac{2 a}{|q|} \frac{\left(q^{2}+2 a B\right)}{R_{1}} \\
& \geqq q^{2} / 4,
\end{aligned}
$$

in view of (2.6) and (2.4). This proves (2.5) Now if $\theta=R_{1}+\frac{2 a}{|q|},(2.5)$ gives

$$
\begin{aligned}
\tilde{u}(\theta) & =\tilde{u}\left(R_{1}\right)+\int_{R_{1}}^{\theta} \tilde{u}^{\prime}(t) d t \\
& \leqq \frac{2 a}{|q|} \cdot \frac{q}{2}=-a .
\end{aligned}
$$

But in the region $u \leqq-a, \tilde{f}(u) \equiv 0$ so $\left(r^{n-1} \tilde{u}^{\prime}\right)=0$ Thus for $r>\theta, r^{n-1} \tilde{u}^{\prime}(r)$ $=\theta^{n-1} \tilde{u}^{\prime}(\theta)<0$, so $\tilde{u}^{\prime}(r)<0$ if $r>\theta$ It follows that $\tilde{u}^{\prime}(r)<0$ if $r \geqq R_{1}$, and the proof is complete.

We can now complete the proof of Theorem 2.2. Let $\varrho(\Omega)$ be as defined in (2.1), and assume $\Omega \subset B_{R}(p)$ for some $p \in \mathbb{R}^{n}$, where $R$ is minimal ${ }^{2}$ By assumption, we have pairs $\left(u_{i}, R_{i}\right), i=1,2$, such that $u_{1}$ and $u_{2}$ are solutions of (1.1), $u_{1}\left(R_{1}\right) \leqq 0, u_{2}\left(R_{2}\right)>u_{1}(0), u_{1}^{\prime}\left(R_{1}\right)<0$, where $0<R_{1} \leqq \varrho(\Omega)$, and $R_{2}>R_{1}$.

Define $\lambda=R_{2} / R$; then $(\lambda \Omega) \subset B_{\lambda R}(p)=B_{R_{2}}(p)$. Also $R_{2} / R_{1} \geqq e(\Omega)$ $=R / \varrho(\Omega)$, so $\lambda \varrho(\Omega)=R_{2} \varrho(\Omega) / R=R_{2} / e(\Omega) \geqq R_{1}$, and hence

$$
R_{1} \leqq \varrho(\lambda \Omega)
$$

Now let $x \in(\lambda \Omega)$; then in view of (2.8), there is some $q \in(\lambda \Omega)$ such that $x \in \overline{B_{R_{1}}(q)} \subset(\lambda \Omega)$. It follows that

$$
x \in \overline{B_{R_{1}}(q)} \subset(\lambda \Omega) \subset B_{R_{2}}(p) \subset B_{2 R_{2}}(q),
$$

since dist $(p, q)<R_{2} ;$ see Figure 3 .

We shall construct our solution by the method of upper and lower solutions; see [14]. To this end we define

$$
u_{0}(x)=\tilde{u}_{1}\left(2 R_{2}\right), \quad x \in(\lambda \Omega),
$$

where $\tilde{u}_{1}$ is obtained from $u_{1}$ according to Lemma 2.3. Since $2 R_{2}>R_{1}$, $\tilde{u}_{1}\left(2 R_{0}\right)<0$, (so $u_{0}$ is not a solution); in fact, $\tilde{u}_{1}<0$ on the complement of

${ }^{2}$ Such an $R$ exists since the function $\psi: \bar{\Omega} \rightarrow \mathrm{R}$ defined by $\psi(q)=\min \left\{R: B_{R}(q)\right\rangle$ $\Omega\}$ is continuous and $\bar{\Omega}$ is compact. 


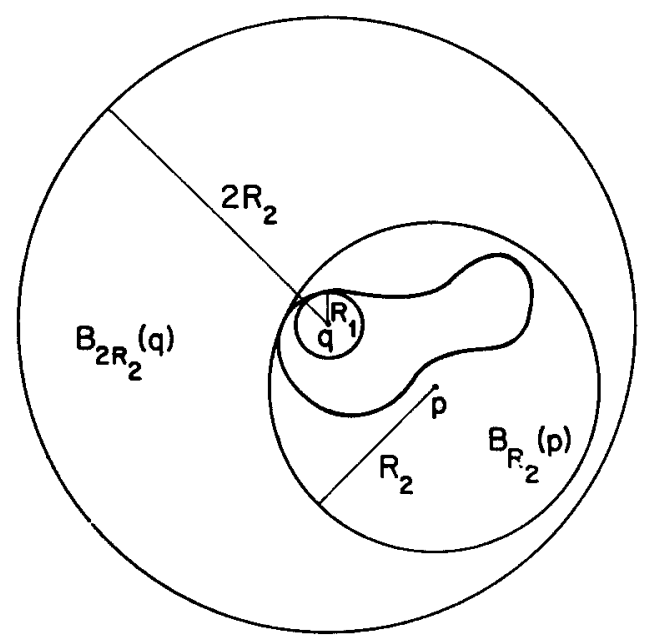

Fig. 3

$B_{R_{1}}(q)$. It follows that $u_{0}$ is a lower solution to

$$
\begin{gathered}
\Delta u(x)+\tilde{f_{1}}(u(x))=0, \quad x \in(\lambda \Omega) \\
u(x)=0, \quad x \in \partial(\lambda \Omega)
\end{gathered}
$$

(where $\tilde{f}$ is obtained from $f$ according to Lemma 2.3).

To construct an upper solution to (2.9), (2.10), we define

$$
U(x)=u_{2}(|x-p|), \quad x \in(\lambda \Omega) \subset B_{R_{2}}(p) .
$$

Then $U$ satisfies (2.9), and for $x \in \partial(\lambda \Omega), U(x)=u_{2}(|x-p|)>u_{1}(0)>0$. We thus see that $U$ is an upper solution (and not a solution), to the problem (2.9), (2.10). Hence by a well-known theorem ([14, Ch. 10]), the problem (2.9), (2.10) has solutions $u$ satisfying

$$
u_{0}(x) \leqq u(x) \leqq U(x), \quad x \in(\lambda \Omega)
$$

Let $\bar{u}$ be a maximal solution with respect to the ordering in (2.11).

To complete the proof, it suffices to show that

$$
\bar{u}(x)>0 \quad \text { if } x \in(\lambda \Omega) .
$$

For, if this were true, then since $\tilde{f}(u)=f(u)$ for $u \geqq 0, \bar{u}$ would be a positive solution of (1.1).

To prove (2.12), let $x_{0} \in(\lambda \Omega)$. Then as above, there is some $q \in(\lambda \Omega)$ with

$$
x_{0} \in B_{R_{1}}(q) \subset(\lambda \Omega) \subset B_{R_{2}}(p) \subset B_{2 R_{2}}(q) .
$$

Define

$$
w(x)=\tilde{u}_{1}(|x-q|), \quad x \in(\lambda \Omega) .
$$


We shall show that $w$ satisfies the following three properties:

(1) $u_{0}(x) \leqq w(x) \leqq U(x), \quad x \in(\lambda \Omega)$;

(2) $w$ is a lower solution of (2.9), (2.10);

(3) $w\left(x_{j}\right)>0$.

If these hold, then from (1) and (2), there is a solution $\overline{\bar{u}}$ of (2.9), (2.10) with $u_{0} \leqq w \leqq \overline{\bar{u}} \leqq U$ on $\lambda \Omega$. Thus by the maximality of $\bar{u}, \bar{u} \geqq \overline{\bar{u}}$ on $\lambda \Omega$, so that using (3), $\bar{u}\left(x_{0}\right) \geqq \overline{\bar{u}}\left(x_{0}\right) \geqq w\left(x_{0}\right)>0$.

We now show that properties (1)-(3) hold. First, recall that $w(x)=$ $\tilde{u}_{1}(|x-q|)$ with $|x-q| \leqq 2 R_{2}$. But since $w$ is a decreasing function, (Lemma 2.3), it follows that $w(x) \geqq \tilde{u}_{1}\left(2 R_{2}\right)=u_{0}(x)$. Next, since $U(x)=u_{2}(|x-p|)$, for $|x|<R_{2}$, we have $U(x) \geqq u_{2}\left(R_{2}\right)>u_{1}(0)=\tilde{u}_{1}(0) \geqq \tilde{u}_{1}(|x-q|)=w(x)$ if $x \in(\lambda \Omega)$; this proves (1). Now $w$ satisfies (2.9), and since $\tilde{u}_{1}(r)<0$ for $r \geqq R_{1}$, (Lemma 2.3), we see that for $x \in \partial(\lambda \Omega),|x-q| \geqq R_{1}$, so $w(x)=\tilde{u}_{1}(|x-q|)$ $<0$; thus $w$ is a lower solution. Finally, $x_{0} \in B_{R_{1}}(q)$ implies $\left|x_{0}-q\right|<R_{1}$ so $w\left(x_{0}\right)=\tilde{u}_{1}\left(\left|x_{0}-q\right|\right)>0$. Thus (3) holds and the proof is complete

We remark that this theorem can be easily extended to equations of the form $\Delta u(x)+\lambda f(u, x)=0, \quad$ with e.g,$\quad f(u, x)=g(x) h(u)$, where $g(x)>0$ and $H\left(u_{0}\right)>0$ for some $u_{0}>0$. This is done by comparison with the equations $\Delta u=\lambda M h(u)+0$ and $\Delta u+\lambda m h(u)=0$, (where $M$ and $m$ are, respectively, the sup and inf of $g$ in $\bar{\Omega}$ ), and yields existence theorems for some range of $\lambda$; see [3] for related results.

Finally, the reader will notice that our technique is actually to construct an upper solution on a ball contaning $\Omega$, and a lower solution on a ball in $\Omega$. Such a strategy is certainly applicable to more general elliptic operators.

\section{§3. Applications}

In this section we shall illustrate the use of Theorem 2.2. Before doing this, however, it is useful to have the following lemma.

Lemma 3.1. Let $F$ be the primitive of $f$ satisfying $F(0)=0$. Suppose $F(\bar{p})>0$ for some $\bar{p}>0$. Then there exists a function $f_{1}$ with $f_{1}(u) \leqq f(u)$ and an $R>0$ and a non-negative solution $u$ of $\Delta u+f_{1}(u)=0$ on $D_{R}^{n}$ with $u(R) \leqq 0$ and $u^{\prime}(R)<0$. (That is, $u$ is a "lower solution" of the Dirichlet problem (see [13]), satisfying $u^{\prime}(R)<0$.)

Proof. Since $F(\bar{p})>0$, we can find a point $p_{0}>0$ with $F(p)<F\left(p_{0}\right)$ for all $p, \quad 0 \leqq p<p_{0} ;$ e.g. $p_{0}=\inf F^{-1}(F(\vec{p}))$. If $f\left(p_{0}\right)=0$, then the conclusion follows from our result in [13]. If $f\left(p_{0}\right)>0$, we let $f_{1}(u)$ be a $C^{1}$-function satisfying $f_{1}(u)=f(u)$ for $0 \leqq u \leqq p_{0}, f_{1}\left(p_{1}\right)=0$ for some $p_{1}>p_{0}$, and $f_{1}(u)>0$ on $p_{0}<u<p_{1} ; c f$. figure 4. Again using the result in [13], we can find positive solutions $u_{1}$ to $\Delta u+f_{1}(u)=0$ on $D_{R}^{n}$ for some $R>0$, with $u_{1}(R), u_{1}^{\prime}(R)<0$. But then $u_{1}$ restricted to the interval $0 \leqq u<p_{0}$ is the desired lower solution. 


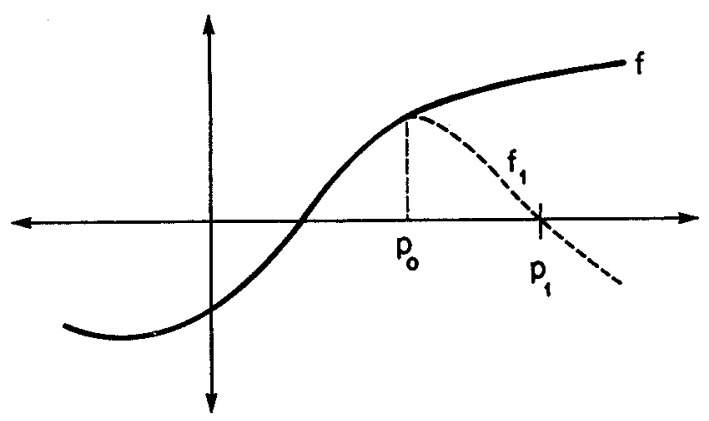

Fig. 4

We remark that the condition $F(p)>0$ for some $p>0$ is a necessary condition for the existence of positive solutions of (1.1), (1.2); see [2]. Thus if $u\left(x_{1}\right)=\max \{u(x): x \in \bar{\Omega}\}$, then $\Delta u\left(x_{1}\right)<0$ so $f\left(u\left(x_{1}\right)\right)>0$. The well-known Pohozaev identity (see [8]),

$$
2 n \int_{\Omega} F(u) d x-(n-2) \int_{\Omega} \mathrm{u} f(u) d x=\int_{\partial \Omega}\left(\frac{d u}{d \vec{n}}\right)^{2}(r \cdot \vec{n}) d s,
$$

implies that $F\left(u\left(x_{1}\right)\right) \geqq \frac{n-2}{2 n} u\left(x_{1}\right) f\left(u\left(x_{1}\right)\right)>0$.

As a first application of Theorem 2.2, we have the following theorem, which extends the main results in [5] and [13].

Theorem 3.2. Let $\Omega \subset \mathbb{R}^{n}$ be a bounded open set with smooth boundary, and suppose that there is a $p_{0}>0$ for which $f\left(p_{0}\right)=0$ and $F\left(p_{0}\right)>0$. Then the conclusion of Theorem 2.2 is valid.

Proof. We shall show that (2.2) holds. Let $\bar{p}=\inf F^{-1}\left(F\left(p_{0}\right)\right)$; then $F(\bar{p})>F(p)$ if $0 \leqq p<\bar{p}$. If $p_{1}=\inf \{p \geqq \bar{p}: f(p)=0\}$, then $f\left(p_{1}\right)=0$, and $F\left(p_{1}\right)>F(p)$ if $0 \leqq p<p_{1}$. From the theorem in [13], there is an $R_{1}>0$ for which there exists a positive radial solution $u_{1}$ to the problem (1.1), (1.2) on the domain $D_{R_{1}}^{n}$, with $u_{1}(0)<p_{0}$ and $u_{1}^{\prime}\left(R_{1}\right)<0$. Choose any $R_{2}>R_{1}$. Setting $u_{2}(x) \equiv p_{0}$ on $D_{R_{2}}^{n}$ and $f_{2}=f$, we see that $N(f) \geqq R_{2} / R_{1}$, for every such $R_{2}$. It follows that $N(f)=+\infty$ and this gives (2.2).

Our next theorem extends to arbitrary domains $\Omega$ a result in [10, Theorem 18]; see also [3].

Theorem 3.3. Let $\Omega$ be a bounded smooth domain. If $f(u) / u \rightarrow 0$ as $u \rightarrow \infty$, and $F\left(p_{0}\right)>0$ for some $p_{0}>0$, then the conclusion of Theorem 2.2 is valid.

Thus for example, $f(u)$ can be as depicted in the figure below, where $k<1$. 


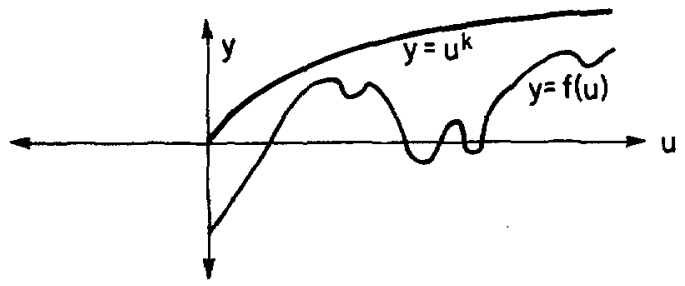

Fig. 5

Proof. If $f\left(p_{0}\right)=0$, then the result follows from the last theorem. If $f\left(p_{0}\right)<0$, then we can find $\tilde{p}<p_{0}$ with $F(\tilde{p})>0$ and $f(\tilde{p})=0$, so this case is reduced to the previous one. Suppose $f\left(p_{0}\right)>0$. Here there are two cases: (i) $f(u)>0$ for all $u>p_{0}$ or (ii) $f(p)=0$ for some $p>p_{0}$. Since case (ii) is easily reduced to the previous ones, we may assume that we are in case (i).

Since $F\left(p_{0}\right)>0$, we may apply Lemma 3.1 to conclude the existence of a non-negative solution $u_{1}$ of $(1.1),(1.2)$ on some ball $D_{R_{1}}^{n}$, with $u_{1}^{\prime}\left(R_{1}\right)<0$. Let $u_{1}(0)=p_{1}>0$. If $\varepsilon>0$ is given, we can find a point $p_{\varepsilon}>0$ such that

$f(u)<\varepsilon^{2} u$ if $u \geqq p_{\varepsilon}$. We may assume that $p_{\varepsilon}>p_{1}$. Now choose $p>2 p_{\varepsilon}$, and let $p_{\varepsilon}<u<p$. Then on this range $F(p)-F(u)=\frac{\varepsilon^{2}}{2}\left(p^{2}-u^{2}\right)$, so with $v=u^{\prime}$, we have $v^{2} \leqq 2 F(p)-2 F(u)=\varepsilon^{2}\left(p^{2}-u^{2}\right)$. If $t(p)$ is defined by $u(t(p), p)=p_{\varepsilon}$ (such a function is easily shown to exist by the methods in [10]), then

$$
\begin{aligned}
t(p) & =\int_{u_{\varepsilon}}^{p} \frac{d u}{-v} \geqq \int_{u_{\varepsilon}}^{p} \frac{d u}{\varepsilon \sqrt{p^{2}-u^{2}}}=\left.\frac{1}{\varepsilon} \sin ^{-1}\left(\frac{u}{p}\right)\right|_{p_{\varepsilon}} ^{p} \\
& =\frac{1}{\varepsilon}\left[\pi / 2-\sin ^{-1}\left(\frac{p_{\varepsilon}}{p}\right)\right] \geqq \frac{\text { const }}{\varepsilon}
\end{aligned}
$$

This shows that $t(p) \rightarrow \infty$ as $\varepsilon \rightarrow 0$. Now as above, we can find solutions $u_{2}(r, p)$ of $(1.1)$ on $0 \leqq r<R_{2}(p)$ with $u_{2}\left(R_{2}(p), p\right)>p_{1}$ where $R_{2}(p)>t(p)$. It follows that $N(f) \geqq t(p) / R_{1}$ so that $N(f)=\infty$ and $(2.2)$ holds.

As a final application, we have the following result (compare with [7, Theorem 2.1]).

Theorem 3.4. Let $f(0)>0$ and assume that $F(p)>0$ for some $p>0$. If $\Omega$ is any bounded domain, the conclusions of Theorem 2.2 are valid.

Proof. From Lemma 3.1, there exists an $R>0$ and a non-negative solution $u_{1}$ of (1.1), (1.2) on $D_{R}^{n}$ with $u_{1}^{\prime}(R)<0$. Since $f(0)>0$, we can find $p_{k} \rightarrow 0$ and solutions $u_{k}$ with $u_{k}(0)=p_{k}$, on $D_{R_{k}}^{n}, R_{k} \rightarrow 0$ (see [10,Th. 18]). Thus $N(f) \geqq R / R_{k}$ so $N(f)=+\infty$ and the result follows. 


\section{§4. The Dirichlet Problem on n-Balls}

We recall the celebrated theorem of GIDAS, Ni \& NIRENBERG [4], which assert. that a positive solution of (1.1), (1.2) on an $n$-ball must be radially symmetrics It thus satisfies the ordinary differential equation

$$
u^{\prime \prime}(r)+\frac{n-1}{r} u^{\prime}(r)+f(u(r))=0, \quad(r=|x|),
$$

and the initial conditions

$$
u(0)=p, \quad u^{\prime}(0)=0,
$$

for some $p>0$. The solution of (4.1), (4.2) will be denoted by $u(r, p)$. We define (cf. [9])

$$
T_{D}(p)=\min \{r>0: u(r, p)=0\},
$$

whenever the set on the right-hand side is not empty. Thus $T_{D}(p)$ is the smallest zero of $u(\cdot, p)$, and if $T_{D}(p)=R$, then $u(\cdot, p)$ is a solution of (1.1), (1.2). In this context, it is convenient to allow the radius $R$ of the $n$-ball $D_{R}^{n}$ to vary with $p$, and we shall thus consider the quantity $p$ as a parameter; see [10-13].

Throughout this section, we shall always assume that $f$ satisfies the following growth condition:

$$
c u^{\alpha} \leqq f(u) \leqq d u^{\beta} \quad \text { for } u \geqq b,
$$

where $\alpha, \beta, c, d$ and $b$ are positive constants. If we take $p \geqq 2 b$, then $f(u)$ is bounded away from zero on the intervals $[p / 2, p]$, and $[b, p]$, so there are numbers $\tau=\tau(p)$, and $T=T(p)$ that satisfy ${ }^{3}$

$$
u(\tau(p), p)=p / 2, \quad u(T(p), p)=b .
$$

Whenever (4.4) holds, we may define the quantity $q=q(p)$ by

$$
q(p)=u^{\prime}(T(p), p) .
$$

Now as we have shown in [10, Theorem 8], in order to prove the existence of positive solutions to the Dirichlet problem, it suffices to show that

$$
\lim _{p \rightarrow \infty} q(p) T(p)=-\infty \text {. }
$$

(In the appendix to this section we give a simpler proof of this result.) If (4.6) holds, then in fact we can find a point $p_{0}>0$ such that if $p \geqq p_{0}$, the function $u(r, p)$ solves (1.1), (1.2) with $R=T_{D}(p)$.

In order to prove (4.6), we write

$$
-q T=-q T^{n-1} / T^{n-2}
$$

and we estimate separately the numerator and denominator in terms of powers of $p$. We then collect these powers, and if the resulting exponent is positive, (4.6) holds and so positive solutions exist. We need a few lemmas before giving our main results (Theorems 4.6, 4.8 and 4.9 , below).

${ }^{3}$ In [10] it is shown that such numbers exist. 
Lemma 4.1. $-q T^{n-1} \geqq$ const. $p^{\alpha} \tau^{n}$.

Proof. From (4.1), we may write $-\left(r^{n-1} u^{\prime}\right)^{\prime}=r^{n-1} f(u)$; thus integrating from $r=0$ to $r=T(p)$ gives

$$
\begin{aligned}
-q T^{n-1} & =\int_{0}^{T} r^{n-1} f(u(r)) d r \\
& \geqq \int_{0}^{T} r^{n-1} c u^{\alpha} d r \\
& \geqq c \int_{0}^{\tau} r^{n-1} u^{\alpha} d r \\
& \geqq c \int_{0}^{\tau} r^{n-1}(p / 2)^{\alpha} d r=\frac{c}{n} \frac{\tau^{n}}{2^{\alpha}} p^{\alpha} .
\end{aligned}
$$

Next we estimate $\tau(p)$.

Lemma 4.2. There are positive constants $k_{1}$ and $k_{2}$ such that

$$
k_{1} p^{1-\beta} \leqq \tau(p)^{2} \leqq k_{2} p^{1-\alpha} .
$$

Proof. For $u \geqq b$, we have

$$
c r^{n-1} u^{x} \leqq-\left(r^{n-1} u^{\prime}\right)^{\prime}=r^{n-1} f(u) \leqq d r^{n-1} u^{\beta}
$$

But $p / 2 \leqq u \leqq p$ when $0 \leqq r \leqq \tau$; thus on this range

$$
c r^{n-1}(p / 2)^{\alpha} \leqq-\left(r^{n-1} u^{\prime}\right)^{\prime} \leqq d r^{n-1} p^{\beta} \text {. }
$$

If we integrate this from 0 to $r \leqq \tau$, we get

$$
\frac{c}{2^{\alpha} n} p^{\alpha} r \leqq-u^{\prime}(r) \leqq \frac{d}{n} p^{\alpha} r
$$

Now integrate this from $r=0$ to $r=\tau$; this gives

$$
\frac{c}{2^{\alpha} n} p^{\alpha} \frac{\tau^{2}}{2} \leqq \frac{p}{2} \leqq \frac{d}{n} p^{\beta} \frac{\tau^{2}}{2},
$$

or

as desired.

$$
\frac{n}{d} p^{1-\beta} \leqq \tau^{2} \leqq \frac{2^{\alpha} n}{c} p^{1-\alpha}
$$

Combining these lemmas gives

$$
\begin{aligned}
-q T & =\frac{-q T^{n-1}}{T^{n-2}} \geqq \text { const. } \frac{p^{\alpha} \tau^{n}}{T^{n-2}} \\
& \geqq \frac{\text { const. }}{T^{n-2}} p^{\alpha}\left(p^{(1-\beta) / 2}\right)^{n},
\end{aligned}
$$


or

$$
-q T \geqq \frac{\text { const. }}{T^{n-2}} p^{(2 \alpha+n-n \beta) / 2} .
$$

We turn our attention now to the problem of estimating $T=T(p)$ from above. This will follow as a consequence of estimates obtained for linear equations, together with a simple monotonicity theorem.

Lemma 4.3. Let $u$ be a positive solution of the (linear) equation

$$
u^{\prime \prime}(r)+\frac{n-1}{r} u^{\prime}(r)+\lambda u(r)=0, \quad 0<r<S(\lambda), \quad \lambda>0,
$$

together with the boundary conditions $u(0)=p, \quad u^{\prime}(0)=u(S(\lambda))=0$. Then $S(\lambda)=\lambda^{-\frac{1}{2}} S(1)$.

Proof. This statement follows from a simple scaling argument. Thus, defining $w(r)=u(c r)$, where $c$ is a constant, we have $w^{\prime}(r)=c u^{\prime}(r), w^{\prime \prime}(r)=c^{2} u^{\prime \prime}(r)$ so if $c^{2}=\lambda^{-1}$,

$$
\begin{aligned}
w^{\prime \prime}(r)+\frac{n-1}{r} w^{\prime}(r)+w(r) & =c^{2} u^{\prime \prime}(c r)+\frac{n-1}{c r} c^{2} u^{\prime}(c r)+u(c r) \\
& =c^{2}\left[u^{\prime \prime}(c r)+\frac{n-1}{c r} u^{\prime}(c r)+\lambda u(c r)\right] \\
& =0 .
\end{aligned}
$$

Also, $w^{\prime}(0)=0, w(0)=p$, so $u(S(1))=w(S(1))$, by uniqueness. Thus $u(c S(1))=w(S(1))=u(S(1))=0$ so $c S(1)=S(\lambda)$ and $S(\lambda)=\lambda^{-1 / 2} S(1)$.

We next have the following monotonicity theorem; it's really a variant of the classical Sturm theorem.

Lemma 4.4. Consider the two equations $\left(-r^{n-1} u_{i}^{\prime}\right)^{\prime}=r^{n-1} f_{i}(u)$, together with the conditions $u_{i}^{\prime}(0)=0$, and $u_{i}(0) \in(0, p], i=1,2$. Suppose that $f_{2}(u) / u>$ $f_{1}(w) / w$ whenever $0<u, w<p$. If $R_{i}$ is defined to be the first zero of $u_{i}, i=1,2$, then $R_{1}>R_{2}$.

Proof. Let $w=u_{1} v_{2}-u_{2} v_{1}$, where $v_{i}=u_{i}^{\prime}, \quad i=1,2$. Then

$$
\left(r^{n} w\right)^{\prime}=r^{n-1} u_{1} u_{2}\left\{\frac{f_{1}\left(u_{1}\right)}{u_{1}}-\frac{f_{2}\left(u_{2}\right)}{u_{2}}\right\}<0,
$$

if $0<r<\min \left(R_{1}, R_{2}\right)$. Now if $R_{2} \geqq R_{1}$, we can integrate (4.8) from $r=0$ to $r=R_{1}$ to get

$$
0>R_{1}^{n-1}\left(u_{1}\left(R_{1}\right) v_{2}\left(R_{1}\right)-u_{2}\left(R_{1}\right) v_{1}\left(R_{1}\right)\right)=-R_{1}^{n-1} u_{2}\left(R_{1}\right) v_{1}\left(R_{1}\right) .
$$

On the other hand $R_{2} \geqq R_{1}$ also implies that $u_{2}\left(R_{1}\right) \geqq 0$, and since $v_{1}\left(R_{1}\right) \leqq 0$, the above inequality is violated. Thus $R_{1}>R_{2}$ as desired. 
We are now able to estimate $T(p)$ from above. We distinguish two cases: $\alpha \leqq 1$ and $\alpha>1$.

Lemma 4.5. Suppose that $f(u) \geqq c u^{\alpha}$ for $u \geqq b$, where $\alpha \leqq 1$, and $c>0$. Then $T(p) \leqq$ const. $p^{(1-\alpha) / 2}$.

Proof. Fix $p>b$ and define $f_{1}(u)=c p^{\alpha-1} u$. Let $f_{2}(u)$ be a $C^{1}$-function for which $f_{2}(u)=f(u)$ if $u \geqq b$, and which also satisfies the inequality $f_{2}(u) / u>c p^{\alpha-1}$ on $0 \leqq u \leqq b$. Then if $b \leqq u<p$, and $0 \leqq w<p$, we have

$$
\frac{f_{2}(u)}{u}=\frac{f(u)}{u} \geqq c u^{\alpha-1}>c p^{\alpha-1}=\frac{f_{1}(w)}{w},
$$

while if $0 \leqq u \leqq b$, and $0 \leqq w<p$, we have

$$
\frac{f_{2}(u)}{u}>c p^{\alpha-1}=\frac{f_{1}(w)}{w} .
$$

Thus the hypotheses of the last lemma are satisfied so (in the obvious notation) $R_{1}>R_{2}$. Now from Lemma 4.3 ,

$$
R_{1}=\frac{S(1)}{\sqrt{\lambda}}=S(1) c^{-\frac{1}{2}} p^{\frac{1-\alpha}{2}}
$$

and since $u_{2}\left(R_{2}, p\right)=0$, and $u_{2}(T, p)=u(T, p)=b$, we have $R_{2}>T$. It follows that $T(p)<R_{2}<R_{1}=$ const. $p^{\frac{1-\alpha}{2}}$.

We may now combine Lemmas 4.1 and 4.5 to obtain the following result.

Theorem 4.6. Assume that $\alpha \leqq 1$, and $\beta-\alpha<2 / n$. Then for all sufficiently large $p$, there are positive solutions $u(\cdot, p)$ of $(1.1)$ on $D_{T_{D}(p)}^{n}$. In particular, if $\alpha=\beta \leqq 1$, there are solutions on $D_{T_{D}(p)}^{n}$ for all sufficiently large $p$.

Proof. Using (4.7) and the last lemma, we have

$$
\begin{aligned}
-q T & \geqq \text { const. } p^{(2 \alpha+n-n \beta) / 2} T^{2-n} \\
& \geqq \text { const. } p^{(2 \alpha+n-n \beta) / 2} p^{(n-2)(\alpha-1) / 2} \\
& =\text { const. } p^{[2-n(\beta-\alpha)] / 2},
\end{aligned}
$$

so that $-q T \rightarrow \infty$ as $p \rightarrow \infty$ if $\beta-\alpha<2 / n$.

We now consider the case where $\alpha>1$. Again we shall crudely estimate $T$ from above and use (4.7).

Lemma 4.7. If $f(u) \geqq c u^{\alpha}$ for $u \geqq b$, where $\alpha>1$, and $c>0$, then $T(p)$ is bounded. 
Proof. If $u \geqq b, f(u) / u \geqq c u^{\alpha-1} \geqq c b^{\alpha-1}$. Let $f>b$; then if we define $f_{1}(u)=c b^{\alpha-1} u$, and let $f_{2}$ be a $C^{1}$-function such that $f_{2}(u)=f(u)$ on $u \geqq b$, and which satisfies the inequality $f_{2}(u) / u \geqq c p^{\alpha-1}$ on $0 \leqq u \leqq b$, then Lemma 4.4 , implies that $R_{1}>R_{2}$. Now as in the proof of Lemma 4.5, $R_{2}>T(p)$ and since $f_{1}$ is linear, $R_{1}$ is a constant, independent of $p$. It follows that $T(p) \leqq$ const.

We use this estimate to prove

Theorem 4.8. Let $\alpha>1$, and suppose that $2 \alpha>n(\beta-1)$. Then for all sufficiently large $p$, there are positive solutions $u(\cdot, p)$ of $(1.1)$ on $D_{T_{D}^{(p)}}^{n}$. In particular, if $1<\alpha=\beta<n /(n-2)$ there are solutions on $D_{T_{D}(p)}^{n}$ for all sufficiently large $p$.

Proof. Using (2.7) and the last lemma, we have

$$
-q T \geqq \text { const. } p^{(n+2 \alpha-n \beta) / 2} \text {, }
$$

where the exponent is positive; thus $-q T \rightarrow \infty$ as $p \rightarrow \infty$.

We may combine Theorems 4.6 and 4.8 to obtain the following theorem.

Theorem 4.9. Suppose that $f(u)=O\left(u^{k}\right)$ as $u \rightarrow \infty$, where $0<k<n /(n-2)$. Then there exist positive solutions to the Dirichlet problem on $D_{T_{D}(p)}^{n}$ for all sufficiently large $p$.

Since $1<n /(n-2)$ for all $n \geqq 2$, we obtain at once the following corollary, (which corrects an error in [10; Corollary 15]).

Corollary 4.10. If $f(u)=O(u)$ as $u \rightarrow \infty$, then for every positive integer $n$, there exists a point $p_{n}>0$ such that if $p \geqq p_{n}$, there exist positive solutions to the Dirichlet problem on $D_{T_{D}(p)}^{n}$.

We close this section with the following observation. Namely, if one were able to strengthen Lemma 4.7 to the statement that if $\alpha>1, T(p) \leqq$ const. $p^{\frac{1}{2-n}}$, that is, if we could estimate the rate of decay of $T(p)$, then existence of positive solutions to the Dirichlet problem would hold for all sufficiently large $p$, whenever $n+2>n \beta-2 \alpha$. Thus in particular if $\alpha=\beta=k$, there would be such solutions whenever $0<k<(n+2) /(n-2)$. This would be the optimal result in this generality.

\section{Appendix}

In this section we shall give a short proof of our basic result in [10]; in particular we shall avoid the use of comparison functions and certain tedious estimates.

Theorem. Suppose that $f(u) \geqq m$ for $u \geqq A$. Then the following statements hold: 
(i) For any $p>A$, there is a $T(p)$ with $u(T(p), p)=A$.

(ii) If $q(p)=u^{\prime}(T(p), p)$, then $-q(p) / T(p) \geqq m / n$.

(iii) If $q(p) T(p) \rightarrow-\infty$ as $p \rightarrow \infty$, then there is a $\bar{p}>0$ such that if $u(0) \geqq \bar{p}$, the problem (4.1), (4.2) has a positive solution, with $u^{\prime}>0$.

Proof. We only prove (iii); parts (i), and (ii) are easily proved as in [9].

Choose $B>0$ such that $-B \leqq f(u) \leqq B, 0 \leqq u \leqq A$; then if $F^{\prime}=f$, $F(0)=0$, we have

$$
|F(u)| \leqq B A, \quad 0 \leqq u \leqq A .
$$

Set $H(r)=v(r)^{2} / 2+F(u(r))$; then $H$ decreases on orbits so $v^{2}+2 F(u) \leqq$ $q^{2}+2 F(A)$, if $0 \leqq u \leqq A$. Using $\left(\mathrm{A}_{1}\right)$, we find

$$
v^{2}(r) \leqq q^{2}+4 B A, \quad 0 \leqq u \leqq A
$$

We shall use this inequality to show

$$
v(r, p)<q / 2 \quad \text { for } T(p) \leqq r \leqq T(p)+\frac{2 A}{|q|},
$$

if $-q(p) T(p) \gg 1$; i.e. if $p \gg 1$.

Thus, we have

$$
\begin{aligned}
v^{2} / 2+F(u)-\left(q^{2} / 2+F(A)\right) & =\int_{T}^{r} H^{\prime}(t) d t \\
& =-(n-1) \int_{T}^{r} \frac{v^{2}}{t} d t \\
& \geqq-(n-1) \int_{T}^{r} \frac{q^{2}+4 B A}{t} d t .
\end{aligned}
$$

It follows that

$$
\begin{aligned}
v^{2} & \geqq q^{2}+2(F(A)-F(u))-(n-1) \int_{T}^{r} \frac{q^{2}+4 B A}{t} d t \\
& \geqq q^{2}-4 B A-(n-1) \int_{T}^{r} \frac{q^{2}+4 B A}{T} d t \\
& =q^{2}-4 B A-\frac{(n-1)}{T}\left(q^{2}+4 B A\right)(r-T) \\
& \geqq q^{2}-4 B A-\frac{(n-1)}{T}\left(q^{2}+4 B A\right) \frac{2 A}{|q|}
\end{aligned}
$$

Thus

$$
v^{2} / q^{2} \geqq 1-4 B A / q^{2}-\frac{2 A(n-1)}{|q| T}\left(1+4 B A / q^{2}\right)
$$


But $q T \rightarrow-\infty$ and $-q / T \geqq m / n$ imply that $q^{2} \rightarrow \infty$ as $p \rightarrow \infty$. Thus for large $p$, we see that the last inequality shows that we can make $v^{2} \geqq q^{2} / 4$ on the requisite interval; this implies $\left(A_{3}\right)$.

From this the proof of (iii) follows rather easily; namely, if $\theta=T(p)+2 A /|q|$, (where $p$ is chosen so large as to make $\left(A_{3}\right)$ valid), we have

$$
\begin{aligned}
u(\theta) & =A+\int_{T(p)}^{\theta} v(t) d t \\
& <A+\frac{q}{2} \frac{2 A}{|q|}=0 .
\end{aligned}
$$

Hence $u$ becomes negative on this interval and since $v<q / 2<0$, on this interval, we see that a solution exists.

\section{§ 5. The Neumann Problem}

We shall show here how the methods of the precedingection can be extended to yield monotone solutions of the Neumann (and in fact, any other linear) boundary-value problem. We continue to assume that inequalities (4.3) hold.

Theorem 5.1. Suppose $\Omega$ is an n-ball, and that $f$ satisfies (4.3), where either i) $\alpha \leqq 1$ and $\beta-\alpha<2 / n$, or ii) $\alpha>1$ and $2 \alpha>n(\beta-1)$. Assume in addition there is an $\eta<0$ such that

$$
f(u)<0 \quad \text { if } u \leqq \eta,
$$

and that

$$
\lim _{u \rightarrow \infty} F(u)=\infty \text {. }
$$

Then there is a $p_{0}>0$ such that if $u(0) \geqq p_{0}$, there is a monotone radial solution of the Neumann problem

$$
\begin{gathered}
A u(x)+f(u(x))=0, \quad x \in D_{T_{N}(p)}^{n} \\
d u(x) / d n=0, \quad x \in \partial D_{T_{N}(p)}^{n} .
\end{gathered}
$$

Recall that $u(r, p)$ is the solution of (4.1) and (4.2); here $T_{N}(p)$ is defined by

$$
T_{N}(p)=\min \{r>0: d u(r, p) / d n=0\},
$$

whenever the set on the right is not empty.

Corollary 5.2. If (5.1) and (5.2) hold, and in addition $f(u)=O\left(u^{k}\right)$ as $u \rightarrow \infty$, $0<k<n /(n-2)$, then the conchusion of Theorem 5.1 is valid.

We remark that if $f(u)>0$ for all $u$, then there cannot be any radial solutions to the Neumann problem (5.3) since the $u$-axis would be a repellor for the 
system (equivalent to (4.1)),

$$
u^{\prime}=v, \quad v^{\prime}=-\frac{n-1}{r} v-f(u)
$$

Thus some conditions like (5.1) and (5.2) are necessary.

Proof. There are two steps in the proof; namely

(i) There exists a $p_{1}>0$ such that if $p \geqq p_{1}$, there is a $\tilde{T}(p)$ satisfying $u(\tilde{T}(p), p)=\eta$.

(ii) For $p \geqq p_{1}, u(r, p)$ is a monotone solution of the Neumann problem.

We begin by showing that (ii) holds. As usual, let $H$, the "total energy", be defined by

$$
H(r) \equiv H(v(r), u(r))=F(u(r))+v(r)^{2} / 2, \quad v=u^{\prime}
$$

Then $H^{\prime}=-(n-1) r^{-1} v^{2}$, so that $H$ decreases on orbits $(u(r, p), v(r, p))$ of (5.4), where, as usual, $p=u(0, p)$.

Now let $p>p_{1}$, and suppose $u(\bar{r}, p)>0$, and $H(\bar{r})=F(u(\bar{r}, p))+v(\bar{r}, p)^{2} / 2$. Using (5.2), we can find $\bar{\eta}<\eta$ for which $F(u) \geqq H(\bar{r})+1$, if $u \leqq \bar{\eta}$. Thus, since $H$ is a decreasing function, and $F(u) \leqq H(u, v)$, we see that $u(r, p)>\bar{\eta}$ for all $r \geqq 0$.

Suppose that the function $u(\cdot, p)$ does not yield a solution of the Neumann problem. Then both $u^{\prime}(r, p)<0$, and $\bar{\eta}<u(r, p) \leqq p$, for all $r \geqq 0$. Thus we see that $u(\cdot, p)$ is a bounded, monotone decreasing function and thus has a limit; say

$$
\lim _{r \rightarrow \infty} u(r, p)=u_{1}
$$

Note that $u(\tilde{T}, p)=\eta$ implies that $u_{1}<\eta$, (since $u^{\prime}<0$ ), so that (5.1) gives $f\left(u_{1}\right)<0$. Thus there is a neighborhood of $u_{1}$ on which $f$ is negative; say $\left|u-u_{2}\right|$ $<\delta$ implies $-B \leqq f(u) \leqq-B / 2$, for some $B>0$. Using (5.6), we can find an $R_{1}>0$ such that $u_{1}<u(r, p)<u_{1}+\delta$, if $r \geqq R_{1}$. It follows that $-B \leqq f(u(r, p)) \leqq-B / 2$ if $r \geqq R_{1}$. Using these facts, we have, for $r \geqq R_{1}$,

$$
-\left(r^{n-1} v\right)^{\prime}=r^{n-1} f(u) \leqq-r^{n-1} B / 2,
$$

and integrating from $R_{1}$ to $r$ gives

$$
-r^{n-1} v(r, p)+q_{1} R_{1}^{n-1} \leqq-\frac{r^{n} B}{2 n}+\frac{R_{1}^{n} B}{2 n}
$$

where $q_{1}=v\left(R_{1}, p\right)$. Thus

$$
-v(r, p) \leqq \frac{-q_{1} R_{1}^{n-1}}{r^{n-1}}-\frac{r B}{2 n}+\frac{B R_{1}}{2 n}\left(\frac{R_{1}}{r}\right)^{n-1},
$$

and this shows that $-v(r, p) \rightarrow-\infty$ as $r \rightarrow \infty$. Thus $v(r, p)>0$ for large $r$, so that $v(r, p)$ must vanish somewhere. This is the desired contradiction, and so the proof of statement (ii) is complete. 
To prove (i), we set $\bar{u}=u-\eta$ and then write the equation in terms of $\bar{u}$ :

$$
-\left(r^{n-1} \bar{u}^{\prime}\right)^{\prime}=r^{n-1} \bar{f}(\bar{u})
$$

where $\overline{f(} \bar{u})=f(\bar{u}+\eta)$. Now if $u \geqq b, c f .(4.3)$, we have the bounds

$$
c(\bar{u}+\eta)^{\alpha} \leqq \bar{f}(\bar{u}) \leqq d(\bar{u}+\eta)^{\beta}, \quad \bar{u} \geqq b-\eta .
$$

Since $\eta>0,(\bar{u}+\eta) \leqq \bar{u}$, so $(5.8)$ gives

$$
\bar{f}(\bar{u}) \leqq d \bar{u}^{\beta} \quad \text { if } \bar{u} \geqq b-\eta .
$$

On the other hand, $b-\eta>-\eta$ so we can find a constant $c_{1}, 0<c_{1}<1$ for which $\left(1-c_{1}\right)(b-\eta) \geqq-\eta$. Thus, if $\bar{u} \geqq b-\eta$, $\left(1-c_{1}\right) \bar{u} \geqq\left(1-c_{1}\right)(b-\eta)$

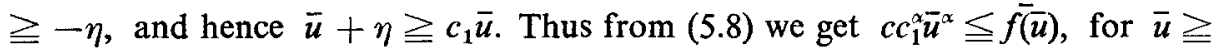
$b-\eta$. Combining this with (5.9), we see that $\overline{f(u)}$ satisfies the same inequalities as does $f(u)$, for large $\bar{u}$. We have seen in $\S 4$ that under the conditions which we have imposed on $\alpha$ and $\beta$, there are radial solutions of the Dirichlet problem. That is, there is a $p_{1}>0$ such that if $p \geqq p_{1}$, there is a $\bar{T}(p)$ for which $\bar{u}(\bar{T}(p), p)$ $=0$; thus $u(\bar{T}(p), p)=\eta$. This proves (i) and completes the proof of the theorem.

We close this section with the observation that any monotone decreasing radial solution to the Neumann problem on an $n$-ball guarantees the existence of a monotone decreasing solution satisfying any linear boundary conditions. Thus the two classical boundary conditions, Dirichlet and Neumann, are actually the most general ones as far as questions of existence are concerned.

Acknowledgement. We thank XABIER GARAIZER for pointing out an error in our original proof of Corollary 4.10 in [10]. We also thank JOHAN DUPONT for showing us the simple proof of Lemma 2.1. This research was supported in part by NSF Contract \# MCS-800-2337. The second author thanks the Mathematics Institute of Aarhus University for supporting this research.

Note added in proof: P. Clément \& G. Sweers (C. R. Acad. Sc. Paris, 302, 681683 (1986)), using similar techniques, have obtained results related to ours in $\S 3$.

\section{References}

1. AmanN, H., Fixed point equations and nonlinear eigenvalue problems in Banach spaces, SIAM Rev., 18, 620-709 (1976).

2. BeRESTYCKI, H., \& P. L. LiONS, Existence of stationary states in nonlinear scalar field equations, in Bifurcation Phenomena in Mathematical Physics and Related Topics, ed. by C. Bardos \& D. Bessis, D. Reidel Publ. Co.: Doredrecht, Boston, London, (1980).

3. Brezis, H., \& L. Oswald, Remarks on sublinear elliptic equations, (preprint).

4. Gidas, B., Ni, W. M., \& L. Nirenberg, Symmetry of positive solutions of nonlinear elliptic equations in $\mathbb{R}^{n}$, Comm. Math. Phys., 68, 209-243 (1975). 
5. Hess, P., On multiple positive solutions of nonlinear elliptic eigenvalue problems, Comm. P.D.E., 6, 951-961 (1981).

6. Hirsch, M. W., Differential Topology, Springer-Verlag: Berlin, Heidelberg, New York, (1976).

7. Lions, P. L., On the existence of positive solutions of semilinear elliptic equations, SIAM, Rev. 24, 441-467 (1982).

8. Nussbaum, R., Positive solutions of nonlinear elliptic boundary-value problems, J. Math. Anal. Appl. 51, 461-482 (1975).

9. Pohozaev, S. I., Eigenfunctions of the equation $\Delta u+\lambda f(u)=0$, Sov. Math. Dok. 5, 1408-1411 (1965).

10. Smoljer, J., \& A. WASSERman, Existence, uniqueness, and nondegeneracy of positive solutions of semilinear elliptic equations, Comm. Math. Phys. 95, 129-159, (1984).

11. Smolier, J., \& A. Wasserman, Symmetry-breaking for positive solutions of semilinear elliptic equations, Arch. Rational Mech. Anal. 95, 217-225 (1986).

12. SMOller, J., \& A. WASSERMAN, Symmetry-breaking for semilinear elliptic equations with general boundary conditions, Comm. Math. Phys. 105, 415-441 (1986).

13. Smoller, J., \& A. Wasserman, An existence theorem for positive solutions of semilinear elliptic equations, Arch. Rational Mech. Anal. 95, 211-216 (1986).

14. Smoller, J., Shock Waves and Reaction-Diffusion Equations, Springer-Verlag: Berlin, Heidelberg, New York, (1983). 